\title{
Anti-Cancer Effects of Radix Angelica Sinensis (Danggui) and N-Butylidenephthalide on Gastric Cancer: Implications for REDD1 Activation and mTOR Inhibition
}

\author{
Kuan-Fu Liao ${ }^{a, b}$ Tsung-Lang Chiuc,d Sung-Ying Huange Teng-Fu Hsiehc,f \\ Shu-Fang Chang ${ }^{g}$ Jhen-Wei Ruan ${ }^{\mathrm{h}}$ Shee-Ping Chen ${ }^{\mathrm{i}}$ Cheng-Yoong Pang ${ }^{\mathrm{j}, \mathrm{k}}$ \\ Sheng-Chun Chiug,l,m
}

aGraduate Institute of Integrated Medicine, China Medical University, Taichung, ${ }^{b}$ Department of Internal Medicine, Taichung Tzu Chi Hospital, Buddhist Tzu Chi Medical Foundation, Taichung, 'School of Medicine, Tzu Chi University, Hualien, dDivision of Neuro-Oncology, Neuro-Medical Scientific Center, Hualien Tzu Chi Hospital, Buddhist Tzu Chi Medical Foundation, Hualien, eDepartment of Ophthalmology, Hsinchu Mackay Memorial Hospital, Hsinchu, 'Department of Urology, Taichung Tzu Chi Hospital, Buddhist Tzu Chi Medical Foundation, Taichung, '9Department of Research, Taichung Tzu Chi Hospital, Buddhist Tzu Chi Medical Foundation, Taichung, Immunology Research Center, National Health Research Institutes, Miaoli, 'Tzu Chi Stem Cells Center, Hualien Tzu Chi Hospital, Buddhist Tzu Chi Medical Foundation, Hualien, 'Department of Medical Research, Hualien Tzu Chi Hospital, Buddhist Tzu Chi Medical Foundation, Hualien, kInstitute of Medical Sciences, School of Medicine, Tzu Chi University, Hualien, 'Department of Laboratory Medicine, Taichung Tzu Chi Hospital, Buddhist Tzu Chi Medical Foundation, Taichung, ${ }^{\mathrm{m} G e n e r a l ~ E d u c a t i o n ~ C e n t e r, ~ T z u ~ C h i ~ U n i v e r s i t y ~ o f ~ S c i e n c e ~ a n d ~ T e c h n o l o g y, ~}$ Hualien, Taiwan

\section{Key Words}

N-butylidenephthalide $•$ Gastric cancer $・$ MTOR $・$ REDD1 $\bullet$ Metastasis

\begin{abstract}
Background/Aims: Radix Angelica Sinensis (danggui in Chinese) is widely used in traditional chinese medicine (TCM). N-butylidenephthalide (BP), a bioactive compound in danggui, is a potential antitumor agent for various cancer types. However, its clinical effect and mechanism in the treatment of gastric cancer remain undetermined. Methods: The in vivo protective effect of danggui in patients with gastric cancer were validated using data from Taiwan's National Health Insurance Research Database (NHIRD). The genes induced by BP-treatment were analyzed by whole transcriptome RNA sequencing (RNA-seq) and validated by real-time

K.-F. Liao and T.-L. Chiu contributed equally to this work.

\begin{tabular}{ll}
\hline Sheng-Chun Chiu & Department of Research, Taichung Tzu Chi Hospital, Buddhist Tzu Chi Medical Foundation \\
& Taichung, 88, Section 1, Fengxing Road, Tanzi Dist., Taichung City 427 (Taiwan) \\
& Tel. +886 4 360606663430, Fax +886 4 25385958, E-Mail honeyhopes@gmail.com
\end{tabular}
\end{abstract}


PCR, western blot and siRNA transfection. The effect of BP on AGS cell migration and invasion was evaluated in transwell assays. The antitumor effects of BP were evaluated in vivo in an AGS xenograft animal model. Results: Danggui users were found to have an increased survival rate when compared with danggui nonusers (log-rank test $p=0.002$ ). The use of danggui highly associated with decreased mortality (the adjusted hazard ratio (HR) of danggui user was 0.72 [95\% CI, 0.57-0.92] $(p=0.009)$. The in vitro results showed that BP inhibited gastric cancer cell proliferation, and triggered cellular apoptosis depending on the activation of mitochondrial apoptotic pathway. Using RNA-seq analysis we found that REDD1 was the highest transcript induced by BP in gastric cancer cells. BP induce an increase of REDD1 expression that inhibits mTOR signaling, thus inhibiting gastric cancer growth. We used RNA interference to demonstrate that the knock-down of REDD1 attenuated the BP-induced mTORC1 activation and growth inhibition. BP suppressed the growth of AGS xenografts tumor in vivo. Conclusion: Danggui can prolong the survival rate of gastric cancer patients in Taiwan. BP caused gastric cancer cell death through the activation of mitochondria-intrinsic pathway and induced the REDD1 expression leading to mTOR signal pathway inhibition in gastric cancer cells. BP inhibited the in vivo growth of AGS xenograft tumors. These results may provide the basis for a new therapeutic approach toward the treatment of gastric cancer progression.

(C) 2018 The Author(s)

Published by S. Karger AG, Basel

\section{Introduction}

Gastric cancer (GC) is the second leading cause of cancer-related death in the world, and the most prevalent cancer in East Asia. In the United States, approximately 21, 000 new cases of gastric cancer were diagnosed in 2010, causing an estimated 10, 570 deaths [1]. Currently radical resectioning of cancerous lesions is the only available cure for gastric cancer, but multi-disciplinary therapy can palliate the disease and even help to prolong life. The treatment options for gastric cancer patients include adjuvant therapy, perioperative chemotherapy, preoperative chemotherapy and combinations of these treatments [2].

Angelica sinensis (Danggui) is a type of traditional Chinese medicine (TCM) widely-used in Taiwan and the prescription of which is covered by Taiwan's National Health Insurance scheme. Taiwan's National Health Insurance Research Database (NHIRD) records the medical history of nearly Taiwan's entire population, and thus provides a means of investigating the incidence of gastric cancer with or without Angelica sinensis exposure. Several studies have used the NHIRD to investigate associations between traditional Chinese medicine and different diseases with high accuracy and validity for population-based research $[3,4]$. Recently, complementary medicine including TCM, have become more widely accepted as potential alternative medicines for cancer treatment. $N$-butylidenephthalide $\left(B P ; \mathrm{C}_{12} \mathrm{H}_{12} \mathrm{O}_{2}\right)$ is a naturally occurring compound isolated from the extract of danggui, and has been reported to exhibit antitumor activity on various types of cancer cell, including glioblastoma multiforme [5, 6], prostate cancer [7, 8], lung cancer [9] and hepatoma [10]. For instance, $\mathrm{BP}$ down-regulates the expression of AP-2 leading to repressing transcriptional activity of hTERT and inhibits the telomerase activity in lung cancer [9]. BP down-regulates the expression of S-phase kinase-associated protein (Skp2) leading to an increase in p16 and p21 expression which cause tumor senescence and cell cycle G0/G1 arrest [5]. BP also modulates the mitochondrial-dependent and ER stress pathway, resulting in cell apoptosis $[7,8]$. These findings indicate that BP is a potential anticancer agent for clinical applications. However, the effect of BP on gastric cancer remains unclear and requires further clarification.

The phosphatidylinositol 3-kinase (PI3K)/Akt/mammalian target of the rapamycin (mTOR) pathway is an important regulation signaling pathway for cell growth, metabolism, survival, metastasis, and resistance to chemotherapy [11, 12]. Genetic alterations in the $\mathrm{PI} 3 \mathrm{~K} / \mathrm{Akt} / \mathrm{mTOR}$ pathway play essential roles in the development and prognosis of gastric cancer $[13,14]$. Various upstream repressors of mTOR signaling have been identified including a 25-kD protein REDD1. REDD1 (regulated in development and DNA damage responses 1), also known as DDIT4, is an mTORC1-dependent signaling pathway inhibitor 
$[15,16]$. It is known to inhibit mTORC1 activity through activation of the TSC1/2 complex [17], and reduction of the phosphorylation of the mTOR substrate: $70-\mathrm{kD}$ ribosomal protein S6 kinase 1 (p70S6K1) and eukaryotic initiation factor 4E binding protein 1 (4E-BP1). The identification of PI3K/Akt/mTOR pathway inhibitors may have the potential to improve the efficacy of gastric cancer treatments.

In this study, we examined the protective effects of danggui and its compound against gastric cancer. To investigate these effects in vivo, we analyzed the survival rate of gastric cancer patients by using data obtained from the NHIRD. To clarify the possible molecular mechanism of BP-induced growth inhibition in gastric cancer, we performed whole transcriptome RNA-seq analysis of BP-treated gastric cancer cells to identify potential transcriptional targets for BP. We found that REDD1 was expressed over 23 fold at $3 \mathrm{~h}$ and 16 fold at $24 \mathrm{~h}$ after BP treatment. We further confirmed the marked induction of REDD1 transcripts and proteins in gastric cancer cell lines. We then demonstrated that REDD1 expression leads to the inhibition of MTORC1 by decreasing the phosphorylation of MTORC1 targets S6K and 4EBP1. The role of REDD1 in BP mediated cell growth and mTOR inhibition was confirmed by REDD1 RNA interference. We then demonstrated that BP suppressed tumor growth with a marked increase in REDD1 and apoptosis-related proteins in tumors by immunohistochemistry in an AGS-xenograft model. Our findings suggest that BP might be a novel anticancer agent for gastric cancer therapy.

\section{Materials and Methods}

\section{Patients and Study Design}

This study used NHIRD records between 1 January, 2003 and 31 December, 2013. In this case-control study, we initially identified patients who had been newly diagnosed with gastric cancer (International Classification of Diseases, Ninth Revision, Clinical Modification (ICD-9-CM) code 151.XX) from inpatient files. This method has been used in other studies to identify gastric cancer patients. The control cohort included one age-matched non-gastric cancer patient for each gastric cancer patient, randomly selected from the same dataset to reduce selection bias by bundling many confounding covariates that may be present in an observational study with this number of variables. Patients who received Chinese herbal products (CHPs) containing danggui between 2003 and 2013 were then identified. The date of initiation of danggui exposure was used as the patient's index date. A total of 32, 279 (11, 203 women and 21, 076 men) patients were included in the study cohort. The danggui non-exposure group included all of other patients not receiving treatment with Chinese herbal products.

\section{Charlson Comorbidity Index Score}

The Charlson Comorbidity Index Score (CCIS) is a widely accepted measure for risk adjustment in administrative claims data sets [18-20]. The CCIS were calculated for each patient by assigning 1 point each for myocardial infarct, congestive heart failure, peripheral vascular disease, dementia, cerebrovascular disease, chronic lung disease, connective tissue disease, ulcer, chronic liver disease or diabetes; assigning 2 points each for hemiplegia, moderate or severe kidney disease, diabetes with end organ damage, tumor, leukemia or lymphoma; 3 points for moderate or severe liver disease; and 6 points each for malignant tumor, metastasis or acquired immune deficiency syndrome.

\section{Other Variables}

The study subjects were classified into three groups by socio-economic status using monthly income as a proxy: (1) low SES, $<$ US\$583, (2) moderate SES, US\$583-833, and (3) high SES, $\geq$ US\$833 [21]. Low income was set at US $\$ 583$ because the NTD equivalent (NTD17500) is the government-stipulated minimum wage for full-time employees in Taiwan in 2009. Geographic region of residence was recorded as northern, central, southern, and eastern Taiwan. 


\section{Cellular Physiology Cell Physiol Biochem 2018;48:2231-2246 \begin{tabular}{l|l|l} 
and Biochemistry & DOI: 10.1159/000492641 & $\begin{array}{l}\text { C } 2018 \text { The Author(s). Published by S. Karger AG, Basel } \\
\text { www.karger.com/cpb }\end{array}$
\end{tabular}}

Liao et al.: Anti-Cancer Effects of Danggui on Gastric Cancer

Cell lines and Cell cultures

The human gastric cancer cell line AGS, NCI-N87 and TSGH-9201 were purchased from BCRC (Bioresource Collection and Research Center, Hsinchu, Taiwan). Each cell line was cultured in its standard medium as recommended by the BCRC. Culture medium, fetal bovine serum, penicillin and streptomycin were all purchased from Invitrogen, Carlsbad, CA, USA. Cell lines were authenticated annually by shorttandem repeat analysis and routinely tested for mycoplasma contamination (BCRC).

\section{Chemicals and reagents}

BP $\left(\mathrm{C}_{12} \mathrm{H}_{12} \mathrm{O}_{2}, 95 \%\right)$ was purchased from Lancaster Synthesis Ltd. (Newgate Morecambe, UK). Cisplatin, Dimethyl sulfoxide (DMSO), [3-(4, 5-dimethyl thizol-2-yl)-2, 5-diphenyl tetrazolium bromide] (MTT), crystal violet, Tween-20, methanol and horseradish peroxidase-conjugated secondary antibodies were purchased from Sigma Chemical Co. (St. Louis, MO, USA). The antibodies were all purchased from Cell Signaling Technology, Inc., (Danvers, MA, USA). Polyvinyldenefluoride (PVDF) membranes, BSA protein assay kit and chemiluminescence reagents were purchased from Amersham Biosciences (Arlington Heights, IL, USA).

\section{RNA isolation, Sequencing and Bioinformatics}

Total RNA was extracted from cell lines using an-RNeasy Mini Kit ${ }^{\circledR}$ (Qiagen, Valencia, CA, USA) and reverse transcribed at $37^{\circ} \mathrm{C}$ for 60 min with an Omniscript RT Kit ${ }^{\circledR}$ (Qiagen) according to the manufacturer's instructions. After RNA extraction, the RNA integrity (RIN Score > 9.0) and quantity was determined using Nanodrop (Thermoscientific, Rochester, USA) and the Agilent 2100 Bioanalyzer (Agilent Technologies, Palo Alto, CA) according to the manufacturer's instructions. Total RNA samples ( $2 \mu \mathrm{g})$ were processed by Beijing Genomics Institute (BGI) for mRNA enrichment by using oligo (dT) magnetic beads. Indexed dsDNA libraries constructed using the Illumina TruSeq RNA sample preparation kit (Illumina, San Diego, CA) were run on a Hiseq2000 for SR50 according to the BGI experiment pipeline. Genes with an adjusted P of less than 0.05 were selected as differentially expressed genes (DEGs) for further gene ontology and pathway enrichment analyses using DAVID [22, 23] and X2K Expression2Kinases software [24] .

\section{Western Blot Analysis}

$5 \times 10^{5}$ cells per 6 -cm petri dish were lysed with $200 \mu$ l M-PER mammalian protein extraction reagent containing protease inhibitor cocktail (Thermo Scientific, Rockford, IL, USA) and centrifuged at 13, $000 \mathrm{xg}$ at $4{ }^{\circ} \mathrm{C}$ for $10 \mathrm{~min}$. The protein concentration in the supernatants was quantified using a BSA Protein Assay Kit. Electrophoresis was performed on a $12 \%$ SDS-PAGE gel in a Mini-PROTEAN Tetra cell electrophoresis System (BioRad, Hercules, USA) using $20 \mu \mathrm{g}$ of protein extract for each lane. Resolved proteins were transferred to PVDF membranes, blocked with $5 \%$ skim milk for $1 \mathrm{~h}$ at room temperature, and finally probed with the specific primary antibodies at $4{ }^{\circ} \mathrm{C}$ overnight. The PVDF membrane was washed three times with TBS $/ 0.2 \%$ Tween- 20 at room temperature and then incubated with the appropriate secondary antibody labeled with horseradish peroxidase (goat anti-mouse or anti-rabbit, 1:10000, Sigma Chemical, St. Louis, MO, USA) for $1 \mathrm{~h}$ at room temperature. All resolved proteins bands were detected using Western Lightning Chemiluminescence Reagent Plus (Amersham Biosciences, Arlington Heights, IL, USA).

\section{Cell Proliferation Assay}

The viability of the cells following treatment with various BP dosages was evaluated using MTT assay preformed in triplicate. Briefly, cells (4 x 104/well) were incubated in 24-well plates containing $0.5 \mathrm{ml}$ of serum-containing medium. Cells were allowed to adhere for 18-24 $\mathrm{h}$ and were washed with phosphatebuffered saline (PBS). Solutions were always prepared fresh by dissolving $0.1 \%$ DMSO (control) or BP in the culture medium before being added to cells. The BP -containing medium was removed after treatment for 24 or $48 \mathrm{~h}$ as indicated. The cells were then washed with PBS, replenished with culture medium containing 300 $\mu \mathrm{g} / \mathrm{ml} \mathrm{MTT}$, and incubated for $1 \mathrm{~h}$ at $37^{\circ} \mathrm{C}$. After the MTT medium was removed, $0.5 \mathrm{ml}$ of DMSO was added to each well. Absorbance at $570 \mathrm{~nm}$ was detected by a multi well plate reader Infinite 200 Pro TecanTM (Tecan, Mannedorf, Switzerland). The absorbance for DMSO-treated cells was considered as $100 \%$. 


\section{Cellular Physiology Cell Physiol Biochem 2018;48:2231-2246 \begin{tabular}{ll|l} 
and Biochemistry Published onlıne: August 15, 2018 & $\begin{array}{l}\text { (c) } 2018 \text { The Author(s). Published by S. Karger AG, Basel } \\
\text { www.karger.com/cpb }\end{array}$
\end{tabular}}

Liao et al.: Anti-Cancer Effects of Danggui on Gastric Cancer

\section{Cell Cycle Analysis}

The cell cycle was determined by flow cytometry following DNA staining to reveal the total amount of DNA. Approximately $5 \times 10^{5}$ of gastric cancer cells were incubated with 50 (AGS) or 75 (NCI-N87 and TSGH9201) $\mu \mathrm{g} / \mathrm{ml} \mathrm{BP}$ for the indicated time. Cells were harvested with trypsin/EDTA, collected, washed with PBS, fixed with cold $100 \%$ ethanol overnight, and then stained with a solution containing $20 \mu \mathrm{g} / \mathrm{ml} \mathrm{PI}, 0.2 \mathrm{mg} /$ $\mathrm{ml}$ RNase A, and $0.1 \%$ Triton X-100 for 30 min in the dark. The cells were then passed through an Accuri C6 flow cytometer to measure the DNA content. The data were obtained and analyzed with CFlow ${ }^{\circledR}$ software.

\section{Cell Migration and Invasion Assay}

Cell migration was determined by the trans-well assays performed using Hanging inserts (Millipore Co., MA, USA) in a 24-well plate. Cells were treated without or with 50 (AGS) or 75 (NCI-N87) $\mu \mathrm{g} / \mathrm{ml} \mathrm{BP} \mathrm{for}$ $24 \mathrm{~h}$, then $5 \times 10^{4}$ cells were seeded in the hanging inserts with serum-free culture medium. Culture medium supplemented with $10 \%$ FBS was added to the bottom chamber. Incubation was carried out at $37^{\circ} \mathrm{C}$ for the indicated time points (migration: $24 \mathrm{~h}$, invasion: $48 \mathrm{~h}$ ). The hanging inserts were washed with PBS, and cells on the upper filter surface were wiped away with a cotton swab. The inserts were subsequently fixed with $10 \%$ formalin for $10 \mathrm{~min}$ at room temperature, stained with $0.2 \% \mathrm{w} / \mathrm{v}$ crystal violet, and washed with PBS; the remaining cells were counted on the opposite side of the filter under a light microscope (Olympus CKX41 fluorescence microscope, Melville, NY, USA) at $200 \mathrm{X}$ magnification. The migration cell numbers of the control group were considered as $100 \%$. For the invasion assay, a Matrigel basement membrane matrix (BD Biosciences, San Jose, CA, USA) was coated to the upper side of the hanging inserts at a concentration of $2 \mathrm{mg} / \mathrm{ml}$. Cells were seeded onto the coated hanging inserts and followed by migration assay protocol.

\section{Real-time RT-PCR Analysis}

Real-time RT-PCR analysis was performed in triplicate in a Step One Plus Real-Time PCR system (Applied Biosystems, Foster City, CA, USA) with Power SYBR ${ }^{\circledR}$ Green PCR Master Mix (Applied Biosystems) in a final volume of $20 \mu \mathrm{l} /$ reaction. Threshold cycle $(\mathrm{Ct})$ value of each tested gene was normalized to the $\mathrm{Ct}$ value of the GAPDH control from the same RNA preparation. The ratio of transcription of each gene was calculated as 2-( $\Delta \mathrm{Ct})$, where $\Delta \mathrm{Ct}$ is the difference $\mathrm{Ct}($ test gene)-Ct(GAPDH). Real-time RT-PCR primer sequences used in this study were: N-cadherin F-5'-ACAGTGGCCACCTACAAAGG-3', R-5'-CCGAGATGGGGTTGATAATG-3', E-cadherin F-5'-ACGTCGTAATCACCACACTGA-3', R-5'-TTCGTCACTGCTACGTGTAGAA-3', CDK1 F-5'CACATGAGGTAGTAACACTCTG-3',R-5'-ATGGTGCCTATACTCCA-3',CyclinB1 F-5'-CGAAGATCAACATGGCAGG-3', R-5'-CTTGGAGAGGCAGTATCAACC-3', Cyclin E F-5'- TTTGCAGGATCCAGATCAAGA-3', R-5'CACAGACTGCATTATTGTCCCAAG-3', CDC25A F-5'-TAAGACCTGTATCTCGTGGCTG-3', R-5'CCCTGGTTCACTGCTATCTCT-3', GAPDH F-5'-CCATGGAGAAGGCTGGGG -3', R-5'-CAAAGTTGTCATGGATGACC -3 .

\section{Animal Studies}

Ethics Statement: The animal use protocol listed below has been reviewed and approved by Institutional Animal Care and Use Committee (IACUC), Hualien Tzu Chi Hospital, approval No: 104-02. All procedures were performed in compliance with the standard operating procedures and relevant guidelines of the Tzu Chi University Laboratory Animal Center (Hualien, Taiwan). Tumors were generated as described previously [25].

\section{Statistical Analysis}

All data are shown as mean \pm S.D. Statistical differences were analyzed using Student's t-test for normally distributed values and by nonparametric Mann-Whitney U-test for values with a non-normal distribution. Significant differences between groups were evaluated using analysis of variance (ANOVA) with Games-Howell test as post-hoc test. 


\section{Results}

Protective effect of danggui in gastric cancer patients from Taiwan

A total of 32,279 gastric cancer patients were selected from NHIRD records from January 1, 2003 to December 31, 2013. Table S1 shows the number of patients, age, gender, and distribution of various comborbidities. After 11 years follow-up, the survival rate analyses demonstrated a strong association between the use of danggui and survival (log-rank test $p=0.002$, Fig. S1). For all supplemental material see www.karger.com/ doi/10.1159/000492641. Using the multivariate Cox model controlling for age, gender, income, urbanization, Charlson comorbidity index (CCIS) and region, the use of danggui remained highly associated with decreased mortality (1:4 matched cohort, the adjusted HR of danggui user was 0.72 ; $95 \%$ CI, 0.57-0.92 [ $p=0.009]$ ) (Table 1 ).

\section{BP inhibited survival rates of human gastric cancer cells}

Table 1 shows the protect effect of danggui for the gastric cancer patients in Taiwan. $\mathrm{N}$-butylidenephthalide (BP), one of the major components found in the chloroform extract fraction of danggui, shown to possess anticancer activity against various human cancer cells. To determine the cytotoxicity effect of BP on gastric cancer cells, 3 human gastric cancer cell lines were treated with increasing concentrations of BP for 24 and $48 \mathrm{~h}$, and subsequently evaluated for cell viability using the MTT assay. As shown in Fig. 1A, all 3 gastric cancer cell lines showed obvious cell rounding up and shrinkage at $48 \mathrm{~h}$ after BP treatment as compared to the untreated cells. The MTT assay further demonstrated that BP significantly decreased the viability of various gastric cancer cell lines in a dose- and time-dependent manner (Fig. 1B). Treatment of AGS cells with 50 or $75 \mu \mathrm{g} / \mathrm{ml}$ BP for $48 \mathrm{~h}$ resulted in respective cell survival rates of $49 \%$ and $24.1 \%$. Treatment of NCI-N87 cells with 50 or $75 \mu \mathrm{g} / \mathrm{ml} \mathrm{BP}$ for 48 $\mathrm{h}$ resulted in respective cell survival rates of $65.2 \%$ and $44.1 \%$. Treatment of TSGH-9201 cells with 50 or $75 \mu \mathrm{g} / \mathrm{ml} \mathrm{BP}$ for $48 \mathrm{~h}$ resulted in respective cell survival rates of $84.3 \%$ and $64.6 \%$. The $\mathrm{IC}_{50}$ at $48 \mathrm{~h}$ of BP treatment in gastric cancer cells were as follows: AGS, $51.9 \mu \mathrm{g} /$ $\mathrm{ml}$; NCI-N87, $69.6 \mu \mathrm{g} / \mathrm{ml}$; and TSGH-9201, $84.1 \mu \mathrm{g} / \mathrm{ml}$. Accordingly, we used 50 (AGS) and 75 (NCI-N87 and TSGH-9201) $\mu \mathrm{g} / \mathrm{ml}$ BP in the subsequent experiments.

\section{Transcriptome analysis of BP-treated human gastric cancer cells}

To understand how BP inhibits proliferation and promotes cell death in human gastric cancer cells, we treated AGS cells with $50 \mu \mathrm{g} / \mathrm{ml}$ BP for 3 or $24 \mathrm{~h}$ and performed global profiling of the transcriptome of BP-treated cells with RNA-seq to identify the candidate genes induced by BP treatment. In RNA-seq analysis, we found 630 significantly up-regulated and 536 significantly down-regulated differentially-expressed genes (DEGs) in BP-treated cells compared with control cells. These DEGs were further investigated using Gene Ontology (GO) enrichment analysis, with biological processes pulled out as being significantly enriched shown in Fig. S1. We found that BP-treated cells had 15 enriched biological processes from the up-regulated genes (Fig. S2A) and 44 enriched biological processes from the downregulated genes (Fig. S2B). Intriguingly, enriched up-regulated biological processes in BP- 
treated cells are mostly involved in metabolism (e.g., carboxylic acid and organic acid transport) and cell death (e.g., cell morphogenesis, proliferation, induction of apoptosis and programmed cell death) while the enriched down-regulated biological processes in BPtreated cells are mostly related to cell cycle and nucleic acid processing. To further pull out potential biological pathways regulated by BP treatment, we used DAVID software to analyze the DEGs from the comparison of BP-treated cells to control cells by KEGG pathway analysis. We found 12 enriched pathways (with at least 3 DEGs in each pathway) in the up-regulated DEGs (Fig. 1C) and 16 enriched pathways in the down-regulated DEGs from BP-treated cells (Fig. 1D). Among these 16 pathways, the down regulation of the cell cycle pathway is consistent with the rest of our experimental results (Fig. 1E). Real time PCR verifications of some representative genes of the cell cycle including Cyclin B1, CDC25A and Cyclin E were downregulated after BP treatment for $24 \mathrm{~h}$ in gastric cancer cell lines. These data demonstrated that cell cycle inhibition following BP treatment might involve in BP-mediated anti-proliferation effects in gastric cancer cells.

\section{Transcriptome analysis revealed that $B P$ up-} regulates REDD1 expression

that contributes to inhibit mTOR signaling in human gastric cancer cells

Among these 12 up-regulated KEGG pathways (Fig. 1C), we found the mTOR signaling pathway is of great interest. Notably, since REDD1 is the upstream regulator of the mTOR signaling pathway and among the significant differential expressed genes (DEGs), REDD1 was nearly the top transcript at 3 and $24 \mathrm{~h}$ following BP treatment (respectively 23.8 and 16.1-fold of up-regulated expression). We therefore confirmed the expression of REDD1 in human gastric cancer cell lines by using quantitative RT-PCR (qRT-PCR) and western blotting analysis. We founded that BP can increase REDD1 expression in a dose-dependent fashion as high as 13.8-fold in AGS cells, 9.5-fold in NCI-N87 cells and 2.8-fold in TSGH-9201 cells (Fig. 2A). Western blotting showed that BP induced REDD1 protein expression as early as

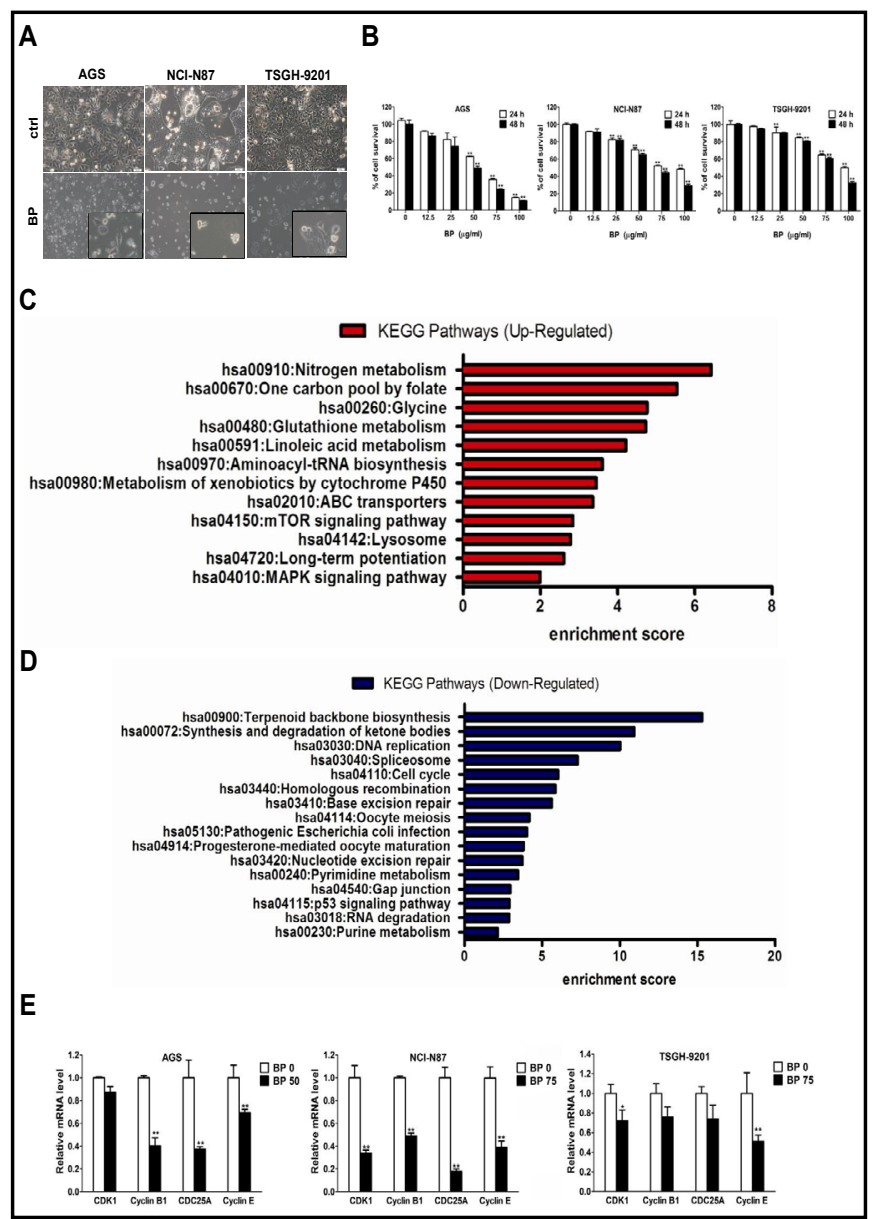

Fig. 1. Effects of BP on human gastric cancer cells. [A] Human gastric cancer cells (AGS, NCI-N87 and TSGH-9201) were treated with $0.2 \%$ DMSO as control or $75 \mu \mathrm{g} / \mathrm{ml}$ BP for $48 \mathrm{~h}$; Scale bar: 50 $\mu \mathrm{m}$. [B] Human gastric cancer cells were treated with increasing concentration of BP (12.5 to $100 \mu \mathrm{g} / \mathrm{ml}$ ) for 24 ( $\square$ ) and $48 \mathrm{~h} \mathrm{(} \mathbf{(})$, respectively, and the survival rate was evaluated with MTT assay. [C] AGS cells were treated with $50 \mu \mathrm{g} / \mathrm{ml}$ BP followed by RNAseq analysis. KEGG pathway analysis of BP up-regulated and down-regulated [D] DEGs. [E] Validation (from qRT-PCR) of the KEGG cell cycle pathway that were significantly inhibited in the down-regulated genes of the comparison of BP-treatment cells to control cells. Data are presented as means \pm S.D. obtained from three different experiments. ${ }^{* *} \mathrm{p}<0.01$ versus vehicle. 
$2 \mathrm{~h}$ after treatment in all human gastric cancer cell lines (Fig. 2B). REDD1 has been shown to inhibit mTORC1 activity through activation of the TSC1/2 complex, and the inhibition of mTORC1 will abrogate the activation of downstream targets by alleviating the phosphorylation of mTOR, p70S6K and 4EBP1. We found that BP also inhibited mTORC1 signaling by decreasing p70S6K and 4EBP1 phosphorylation as early as $2 \mathrm{~h}$ after BP treatment (Fig. 2B). These results demonstrate that BP-induced REDD1 expression and repressed the mTOR signaling pathway in human gastric cancer cell lines.

REDD1 involves in the BPmediated inhibitory effect of gastric cancer cell proliferation

We further used a siRNA approach to determine the role of REDD1 in the anti-proliferation effect of BP in gastric cancer. AGS cells were transfected with siRNAs for REDD1, and then treated with or without $50 \mu \mathrm{g} / \mathrm{ml} \mathrm{BP}$ for $48 \mathrm{~h}$. In western blot analysis, transfection of si-REDD1 suppressed REDD1 expression induced by $\mathrm{BP}$ in AGS cells, as compared with cells transfected with control scrambled siRNA. The expression inhibition of REDD1 restored the phosphorylation of downstream targets including mTOR, p70S6K and 4EBP1 (Fig. 2C). Cell death induced by BP treatment was respectively rescued by 22.4 and $17.3 \%$ in cells transfected with 20 or 40 nM REDD1 siRNA, as

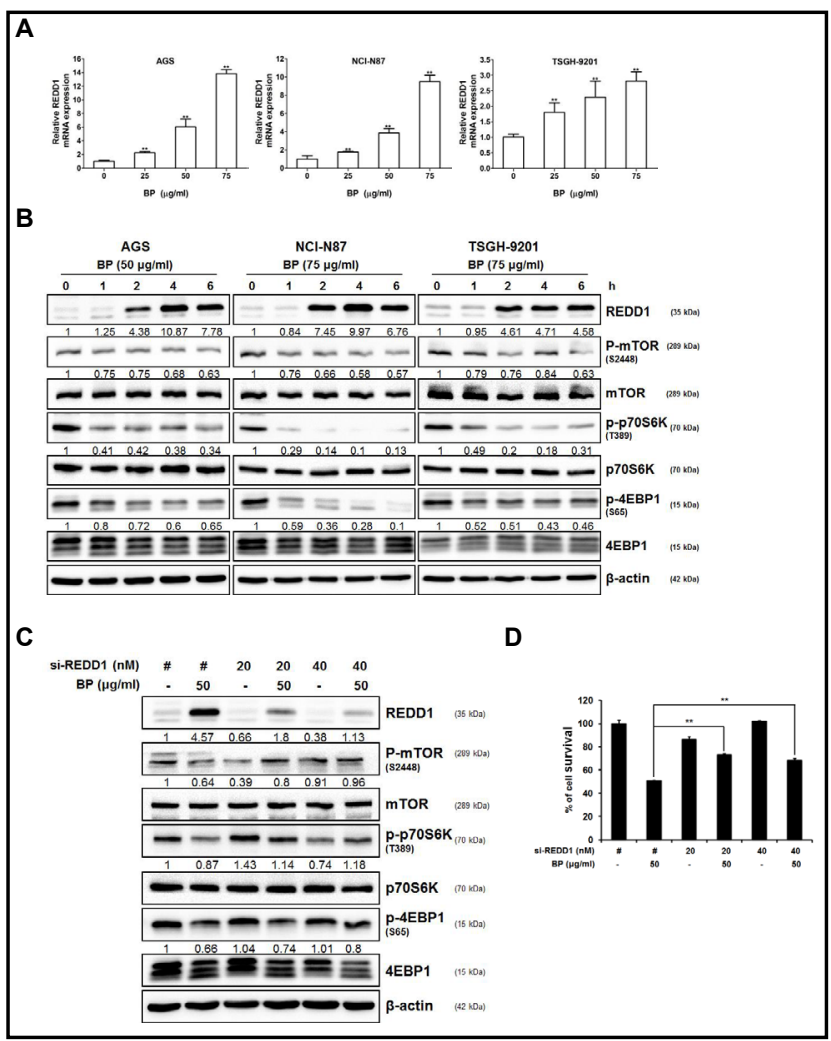

Fig. 2. BP induced REDD1 expression and suppression of the mTOR signaling pathway in human gastric cancer cells. [A] qRT-PCR analysis of REDD1 in human gastric cancer cells treated with increasing concentration of BP (25 to $75 \mu \mathrm{g} / \mathrm{ml}$ ) for $24 \mathrm{~h}$. [B] Human gastric cancer cells were treated with $0.2 \%$ DMSO (control) or BP for indicated time points, and western blot analysis was performed for REDD1 and mTOR signaling pathway-related proteins. $\beta$-actin was used as an internal control. [C] AGS cells were transfected with scramble siRNA (\#), 20, or $40 \mathrm{nM}$ REDD1 siRNA for $48 \mathrm{~h}$ using the RNAifect transfection reagent. After $48 \mathrm{~h}$ treatment with 50 $\mu \mathrm{g} / \mathrm{ml} \mathrm{BP}$, western blot analysis was performed for REDD1 and mTOR signaling pathway-related proteins. [D] BP-induced anti-proliferative activity was measured using MTT assay in AGS cells transfected with scramble siRNA (\#), $20 \mathrm{nM}$ or 40 nM REDD1 siRNA for $48 \mathrm{~h}$ and then treated with $50 \mu \mathrm{g} / \mathrm{ml}$ BP for $24 \mathrm{~h}$. Data are expressed as means \pm S.D. from three independent experiments, ${ }^{* *} \mathrm{p}<0.01$ versus vehicle. compared to scrambled siRNAtransfected cells (Fig. 2D). Taken together, these results indicated that BP-induced cell death might be at least partly due to the induction of REDD1 expression that down-regulated the mTOR signaling pathway.

\section{BP induced apoptotic cell death in human gastric cancer cells}

To explore the underlying mechanism in BP-induced gastric cancer cell death, human gastric cancer cells were treated with BP for 24 or $48 \mathrm{~h}$ and analyzed by flow cytometry (Fig. S3). The appearance of the sub-G1 cell population can be considered to indicate the extent of early apoptotic cell death. As shown in Fig. S3, the sub-G1 population increased to 9.7 


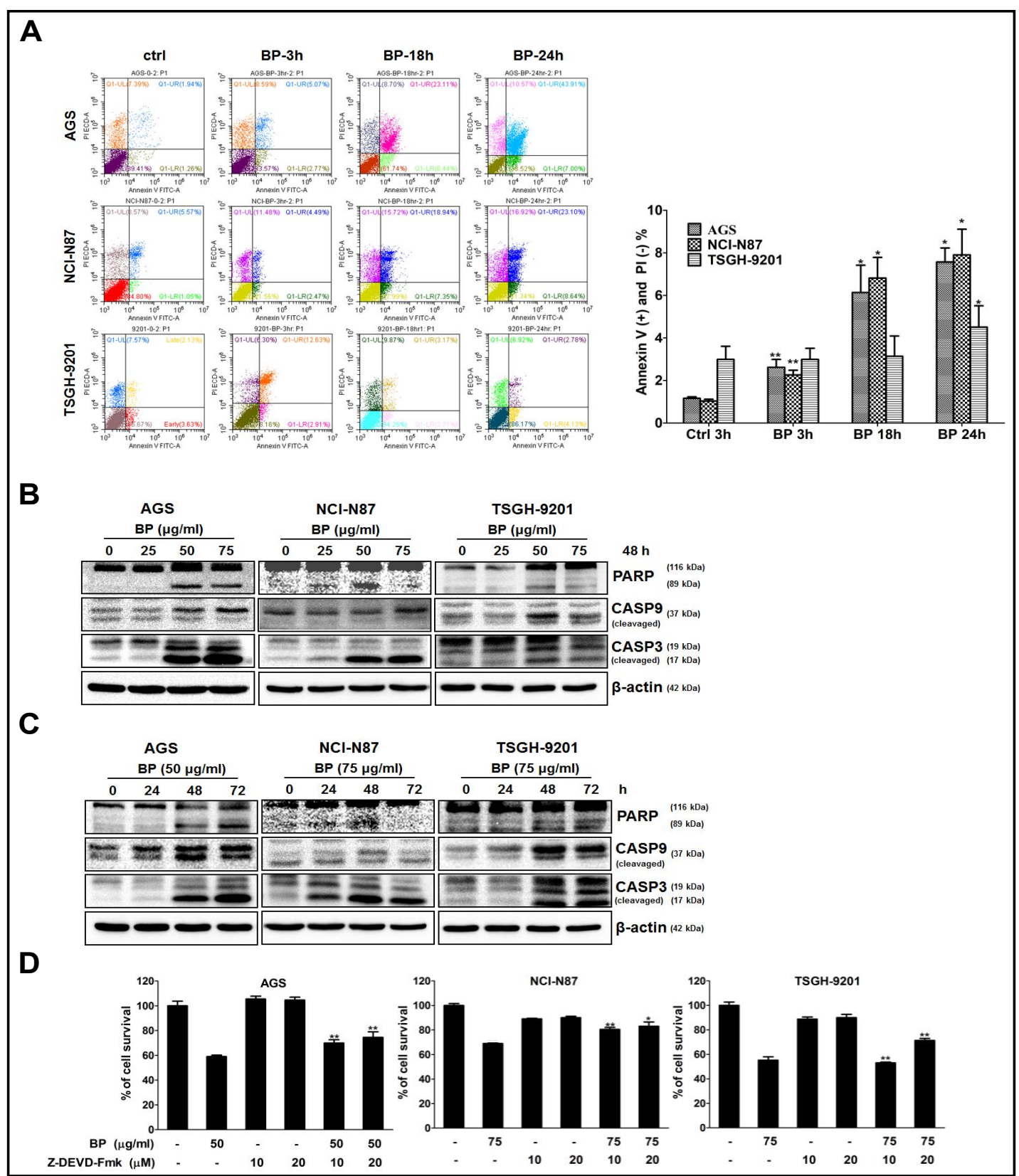

Fig. 3. BP induced mitochondrial-mediated apoptosis in human gastric cancer cells. [A] Human gastric cancer cells were analyzed by Annexin V-FITC staining in the vehicle group (0.2\% DMSO) for $3 \mathrm{~h}$ or at presence of 50 (AGS) or 75 (NCI-N87 and TSGH-9201) $\mu \mathrm{g} / \mathrm{ml}$ BP for 3, 18 and $24 \mathrm{~h}$. The percentage of annexin V-FITC positive and PI negative population in gastric cancer cells is shown. [B] Human gastric cancer cells were treated with 25, 50 and $75 \mu \mathrm{g} / \mathrm{ml} \mathrm{BP}$ for $48 \mathrm{~h}$, and western blot analysis was performed for cleaved PARP, caspase 9 and caspase 3. [C] Human gastric cancer cells were treated with BP for 0, 24, 48, or $72 \mathrm{~h}$, and western blot analysis were performed for cleaved PARP, caspase 9 and caspase 3. [D] Human gastric cancer cells pretreated with caspase 3 inhibitor Z-DEVD-fmk (10 or $20 \mu \mathrm{M})$ were subjected to MTT assay for $1 \mathrm{~h}$ and then treated in the presence or absence of BP for $48 \mathrm{~h}$. The BP-induced cell death was partly blocked after caspase 3 inhibitor pretreatment as compared to the BP alone group. The values are the mean \pm S.D. from three independent experiments, ${ }^{*} \mathrm{p}<0.05$ versus vehicle, ${ }^{* *} \mathrm{p}<0.01$ versus vehicle. 


\section{Cellular Physiology Cell Physiol Biochem 2018;48:2231-2246 \begin{tabular}{l|l} 
DOI: 10.1159/000492641 & and Biochemistry \\
Published online: August 15, 2018 & $\begin{array}{l}\text { O 2018 The Author(s). Published by S. Karger AG, Basel } \\
\text { www.karger.com/cpb }\end{array}$
\end{tabular}}

Liao et al.: Anti-Cancer Effects of Danggui on Gastric Cancer

\% (AGS), 5 \% (NCI-N87), and $6.3 \%$ (TSGH-9201) after BP treatment for 48 h. For further demonstrate the involvement of apoptosis, human gastric cancer cells were treated with BP for indicated time points, and apoptotic cells were accessed by annexin V-FITC staining (Fig. 3A). Apoptosis was noted as early as $3 \mathrm{~h}$ after BP treatment; the PI-negative, annexin V-FITC positive populations denote as early apoptotic cells and increased after BP treatment as compared to the vehicle group (For BP treatment $24 \mathrm{~h}$ : AGS: $7.6 \%$; NCI-N87: $7.9 \%$ and TSGH-9201: $4.5 \%$ ). These results suggested that BP induced apoptotic cell death in human gastric cancer cells.

\section{BP induced mitochondrial-mediated apoptosis in human gastric cancer cells}

Caspase activation plays a central role in the induction of apoptosis. To evaluate whether the growth inhibition induced by BP is due to apoptosis, we determined the crucial apoptotic signal caspase $-9 / 3$ activation in gastric cancer cell lines. Cleavages of caspase -9 and -3 increased dose- and time-dependently in gastric cancer cells after BP treatment (Fig. 3B and C). However, the activation of caspase 8 was not noted (data not shown). These results suggested that gastric cancer cells might undergo mitochondria-mediated apoptosis after exposure to BP. To further demonstrated the role of caspase 3 in BP-induced apoptosis, cells were pretreated with caspase 3 inhibitor Z-DEVD-fmk (10 or $20 \mu \mathrm{M})$ for $1 \mathrm{~h}$ and then treated in the present or absent of BP for $48 \mathrm{~h}$ (Fig. 3D). The BP-induced cell death was partly blocked after caspase 3 inhibitor pretreatment ( $20 \mu \mathrm{M}$ group: $15.6 \%$ in AGS, $14.1 \%$ in NCI-N87, 16.1\% in TSGH-9201) as compared to the BP alone group. These data suggest that BP induced gastric cancer cell apoptosis via the mitochondria-dependent pathway. To a lesser extent, these results further confirm that the caspase-dependent pathway is involved in BP-induced human gastric cancer cell death.

\section{Effect of BP on the migration and invasion of human gastric cancer cells}

Tumor cell malignancy usually correlates to their degree of proliferation and invasion. Trans-well assays were performed to elucidate the effect of BP on the motility of gastric cancer cells. The migration and invasion of BP-treated human gastric cancer cells decreased significantly as compared to the control group in gastric cancer cells (Fig. 4A). The migrated cells accounted for $21.9 \%$ (AGS) and $11.6 \%$ (NCI-N87), as compared to the non-treated cells. The invasive cells accounted for $0.6 \%$ (AGS) and $59.2 \%$ (NCI-N87), as compared to the non-treated cells.

The modulation of epithelial-mesenchymal transition (EMT) is seen as an important target in metastatic regulation. To evaluate whether BP could affect the metastatic potential of gastric cancer cell lines, the expression levels of EMT-related genes were examined using real-time RT-PCR. BP treatment for $24 \mathrm{~h}$ significantly up-regulated the mRNA expressions of E-cadherin (2.1 fold in AGS and 2.2 fold in NCI-N87), whereas down-regulated the expressions of N-cadherin (0.8 fold in AGS and 0.5 fold in NCI-N87) (Fig. 4B). The EMTrelated genes expression was regulated after BP treatment. The up-regulation of epithelial marker E-cadherin is only visible in AGS cells. The down-regulation of mesenchymal markers N-cadherin and Vimentin, transcription factor Slug at the protein level is evidenced by western blot (Fig. 4C). These results suggest that BP is a potential inhibitor of EMT in human gastric cancer cells.

\section{BP suppresses tumor growth of AGS cancer cells in NOD-SCID mice}

To evaluate the antitumor activity of BP in vivo, human gastric cancer xenografts were established by injection of $5 \times 10^{5}$ AGS cells into the dorsal subcutaneous tissue of NOD-SCID mice. As shown in Fig. 5A, the relative tumor volumes in mice treated with 300, 500 and 700 $\mathrm{mg} / \mathrm{kg}$ BP were smaller than the vehicle-treated control mice on Day 31 (48.6 \%, $P=0.02$; $41.9 \%, P=0.01 ; 34.8 \%, P=0.002$, respectively). Tumors in the control and therapeutic groups were removed and photographed (Fig. 5B). Tumor weight was significantly decreased by $36.2 \%(700 \mathrm{mg} / \mathrm{kg}, P=0.007)$ in the BP-treated groups as compared with the control group. Hematoxylin/eosin (H\&E) staining revealed that BP-treated tumor specimen had dark and 
Fig. 4. Effect of $B P$ on the migration and invasion of human gastric cancer cells. [A] Human gastric cancer cells (AGS and NCI-N87) were pretreated with $0.2 \%$ DMSO as control or 50 and $75 \mu \mathrm{g} / \mathrm{ml} \mathrm{BP}$ for $24 \mathrm{~h}$ and then seeded onto the transwell hanging insert coating with (invasion) or without (migration) matrigel for different time points (migration: $24 \mathrm{~h}$, invasion: $48 \mathrm{~h}$ ). Images were captured using an inverted microscope with 200-x magnification; Scale bar: $50 \mu \mathrm{m}$. The migration and invasion of human gastric cancer cells were quantified by enumerating the stained cells that migrated into the underside of the hanging insert membrane. [B] Human gastric cancer cells were treated with 50 or $75 \mu \mathrm{g} / \mathrm{ml} \mathrm{BP}$ for $24 \mathrm{~h}$, and the mRNA expression levels of E-cadherin and $\mathrm{N}$-cadherin were determined by qRT-PCR analysis. c Human gastric cancer cells were treated with 25, 50 and $75 \mu \mathrm{g} / \mathrm{ml}$ BP for $48 \mathrm{~h}$, and western blot analysis was performed for EMT-related genes. Data are presented as means \pm S.D. from three different experiments. * $\mathrm{p}<0.05$ versus vehicle; ${ }^{* *} \mathrm{p}<0.01$ versus vehicle.

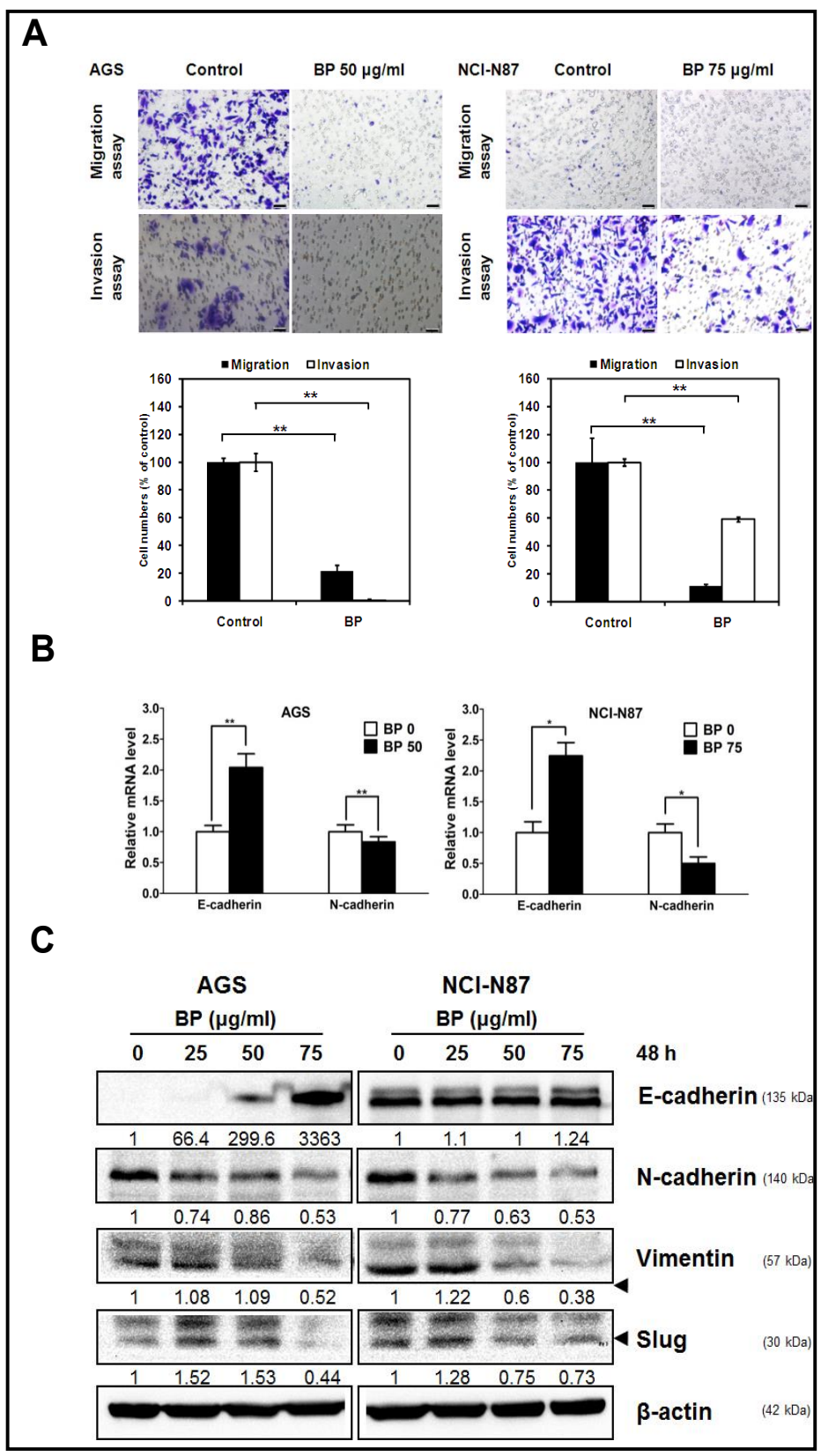

dense nuclei as compared to the control group. In addition, control group tumor specimens had large and irregular nuclei and a large number of signet ring cells which are associated with a poor prognosis (Fig. 5C). Furthermore, immunohistochemistry for the proliferation marker Ki67 showed more markedly increased Ki67 expression in the control group than the BP-treated group (Fig. 5C). Upregulation of REDD1 in the BP-treatment tumor was observed by immunohistochemistry staining (Fig. 5C). Caspase-3 activation was also observed in BP treatment tumors (Fig. 5D). No significant body weight differences were found between the control and BP-treated groups. These results suggest that BP-induced gastric cancer cell death might be correlated with REDD1 expression in vivo. Further tumor formation experiments using the REDD1 knock-down cells are needed to establish a cause-effect relationship. 
Fig. 5. BP inhibits xenographic growth of AGS cells in NOD-SCID mice. [A] Approximately $5 \times 10^{5}$ AGS cells were injected into the dorsal subcutaneous tissue of NOD-SCID mice. When the tumor reached 100-250 $\mathrm{mm}^{3}$, AGS tumor-bearing mice were administrated s.c. with vehicle control or 300 to $700 \mathrm{mg} /$ $\mathrm{kg}$ BP on days 0-4 for 5 days. The relative tumor volumes of the control and the BP-treated groups are shown as means \pm S.D. of tumor volume at each time point. [B] Tumors from the control and therapeutic groups were removed and weighed on Day 31. Average

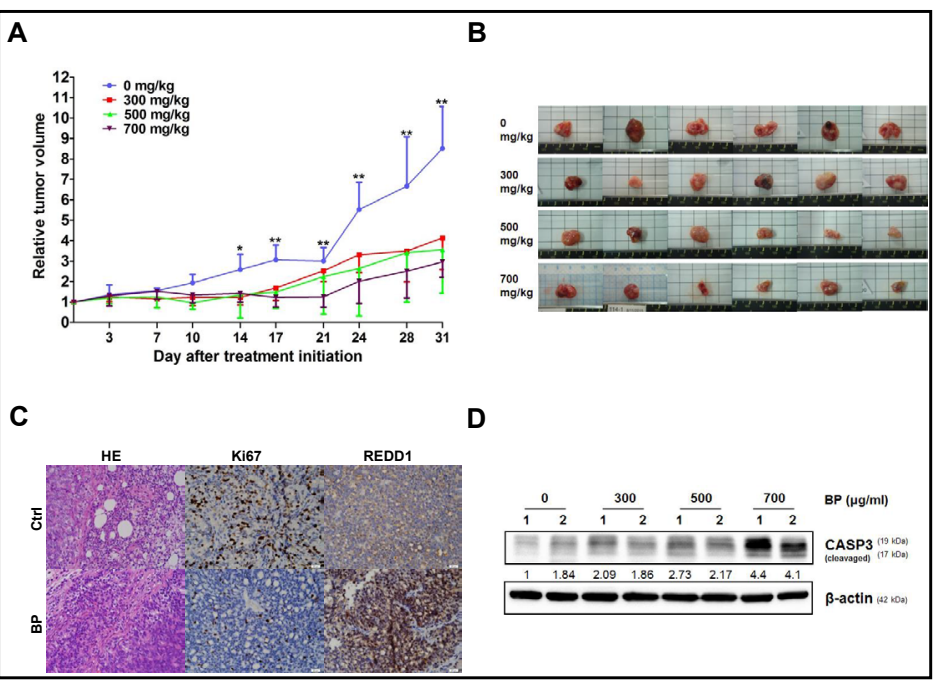
tumor weight from the BP-treated group $(700 \mathrm{mg} / \mathrm{kg})$ was $36.2 \%$ smaller than that of the control group. [C] Tumor tissue sections with HE staining. Ki67 and REDD1 expressions were immuno-histochemically identified in the control and the BP-treated groups. The REDD1-positive cells were stained brown. Expression of active form caspase-3 in the AGS xenograft tumor tissue were upregulated after BP administration as compared to control group by western blotting analysis [D].

\section{Discussion}

The popularity of danggui in Taiwan provides an opportunity to investigate the incidence of gastric cancer with or without danggui exposure through the NHIRD. Given the time and cost required for large-scale clinical trials, the NHIRD presents a reasonable and viable approach for research on herbal and complementary and alternative medicine (CAM). The NHIRD suffers from several limitations including lack of information about patient lifestyle, the severity of the cancer and family history of cancer. However, previous studies have shown that the NHIRD database provides approximately $95 \%$ consistency between patient records and claims data, indicating a high degree of validity and accuracy. A total of 32, 279 patients (both with and without gastric cancer) were selected from NHIRD records from January 1, 2003 to December 31, 2013. Table S1 shows the number of patients, ages, genders, and distribution of various comborbidities. Overall, patients with exposure to danggui displayed a significant decrease in mortality (adjusted HR of danggui user was 0.72; $95 \% \mathrm{CI}$, 0.57-0.92 [ $p=0.009]$ ). Although NHIRD data indicates danggui exhibits a protective effect on gastric cancer patients in Taiwan, serious randomized, double-blind, and placebo-controlled trials are necessary to confirm this finding. In addition, to clarify the relationship between danggui and gastric cancer, we carried out in vitro and in vivo experiments to provide supportive evidence for the therapeutic effects of BP in gastric cancer therapy.

$\mathrm{BP}$ has been reported to prolong the survival of ALS mice by down-regulating autophagy and upregulating mTOR expression [26]. Combining bone marrow-derived mesenchymal stem/stromal cells (BM-MSCs) with two main components of Radix Angelica Sinensis, BP and sodium ferulate (SF), contribute to angiogenesis after cerebral ischemia which is associated with AKT/mTOR signaling activation [27]. Interestingly, BP induced different degrees of mTOR regulation which might explain cell or tissue specificity. Our study demonstrated that BP causes a marked increase in REDD1 expression and inhibits the mTOR signaling pathway. REDD1 plays a major role in the inhibition of mTOR activity but may also play a partial role in growth inhibition induced by BP in gastric cancer cells. The inhibition of REDD1 expression by RNA interference did not completely block the growth inhibitory effect of BP in AGS cells (Fig. 2D). BP also induced cell cycle arrest- and mitochondrial-dependent apoptosis gene 
expression in gastric cancer cells. We have further demonstrated the tumor suppression potential in xenograft animal models. Taken together, these results suggest the therapeutic potential of BP in clinical applications.

REDD1 positively controls caspase-2-dependent cell death by inhibiting mTOR in ovarian cancer cells [28]. The blockade of fatty acid synthase (FASN) induced an ATF4dependent transcriptional induction of REDD1, facilitating caspase-2 dimerization/ activation and leading to ovarian cancer cell death. Interestingly, our RNA-seq data showed that the FASN gene was downregulated 1.4-fold, but ATF4 and caspase- 2 were respectively upregulated 2.4 and 1.17-fold after $24 \mathrm{~h}$ of BP treatment in AGS cells as compared to the control cells. These data suggest that the novel REDD1-mTOR-caspase-2 pathway may be involved in BP-mediated gastric cancer cell death. In addition, REDD1 was reported to mediate mTOR inhibition and cell cycle arrest through the induction of metformin in cancer cells [29]. Similar cell cycle arrest effects were also observed in BP-treated gastric cancer cells demonstrated by real-time PCR. Taken together, REDD1 and mTOR inhibition may be one of several mechanisms involved in BP-mediated gastric cancer growth inhibition.

Previous report identified that REDD1 encodes a shared transcriptional target for p53dependent stress response, as well as in p63-mediated regulation of epithelial differentiation [30]. In addition, suppression of mTORC1 signaling to 4E-BP1 requires the coordinated activity of two tumor suppressors, p53 and p63 [31]. BP was also shown to induce p53 expression and phosphorylation in brain tumor cells [6], which suggests that BP induced REDD1 expression via the p53 and p63 activation, which merits further investigation.

REDD1 was identified in screening for genes that are upregulated by hypoxia [32]. The hypoxia-induced inhibition of mTOR signaling requires REDD1 and TSC1/TSC2 complex [16, $33,34]$. However, this requirement is cell-type specific, for example REDD1 is was not required for hypoxia-induced mTORC1 inhibition in hepatocytes [35]. Moreover, REDD1 ablation had no effect on glucocorticoid-induced mTORC1 inhibition and apoptosis in thymocytes ex vivo [36]. Our results showed that BP increases REDD1 expression and decreases mTOR and p70S6K phosphorylation nearly simultaneously (Fig. 2B). Furthermore, the knock-down of REDD1 only partially attenuated the BP-induced mTORC1 activation and growth inhibition (Fig. 2C). Thus, we cannot rule out the involvement of the REDD1-independent mechanism in BP-induced mTOR inhibition and apoptosis induction.

Gastric cancer genesis and metastasis is closely related to EMT. Several studies have shown that the initiation of gastric cancer and its malignancy are related to E-cadherin mutations $[37,38]$. In addition, $\mathrm{N}$-cadherin expression has been associated with the invasive phenotype of gastric cancer and may override the function of E-cadherin [39]. The levels of N-cadherin, Snail (Snail1), Slug (Snail2), and Vimentin, are all up-regulated in patients with dysplasia or early Gastric cancer, while the level of E-cadherin is decreased in these patients [40]. In the present study, transwell assay results showed BP suppressed the migration and invasion of gastric cancer cells in vitro. Since BP inhibits proliferation and induces apoptosis in gastric cancer cells, we could not exclude the possibility that the results obtained were partly affected by the impact of BP on cell proliferation/apoptosis. BP clearly up-regulated the expression of E-cadherin in AGS cells but not in NCI-N87 cells. BP down-regulated the expression of mesenchymal markers N-cadherin, Slug, and Vimentin, suggesting that BP is a potential agent to control and inhibit EMT in gastric cancer cells.

\section{Conclusion}

The present study demonstrated that danggui has protective effects for gastric cancer patients in Taiwan. BP inhibits proliferation and induces mitochondrial-dependent apoptosis in gastric cancer cells. BP suppresses mTOR signaling through up-regulated REDD1 expression. BP also inhibits the migration and invasion of gastric cancer cells through modulated the EMT. BP suppresses the growth of AGS xenografts tumor in vivo. Together, these results suggest that BP might be a potential candidate for gastric cancer therapy. 


\section{Cellular Physiology Cell Physiol Biochem 2018;48:2231-2246 \begin{tabular}{ll|l} 
DOI: 10.1159/000492641 & $\begin{array}{l}\text { O 2018 The Author(s). Published by S. Karger AG, Basel } \\
\text { www.karger.com/cpb }\end{array}$
\end{tabular}}

\section{Acknowledgements}

This work was supported by grants from the Taichung Tzu Chi Hospital, Buddhist Tzu Chi Medical Foundation, Taichung, Taiwan (TTCRD103-12, TTCRD104-04, TTCRD104-10, TTCRD104-19). The funders had no role in study design, data collection and analysis, decision to publish, or preparation of the manuscript. We thank Hsin-Rong Wu for the assistance in animal studies. This work was supported by grants from the Taichung Tzu Chi Hospital, Buddhist Tzu Chi Medical Foundation, Taichung, Taiwan

\section{Disclosure Statement}

The authors declare that they have no conflict of interests.

\section{References}

1 Jemal A, Siegel R, Xu J, Ward E: Cancer statistics, 2010. CA Cancer J Clin 2010;60:277-300.

$\checkmark 2$ Jiang Y, Ajani JA: Multidisciplinary management of gastric cancer. Curr Opin Gastroenterol 2010;26:640646.

-3 Wu CY, Yang YH, Lin YY, Kuan FC, Lin YS, Lin WY, Tsai MY, Yang JJ, Cheng YC, Shu LH, Lu MC, Chen YJ, Lee KD, Kang HY: Anti-cancer effect of danshen and dihydroisotanshinone I on prostate cancer: targeting the crosstalk between macrophages and cancer cells via inhibition of the STAT3/CCL2 signaling pathway. Oncotarget 2017;8:40246-40263.

-4 Lin YY, Lee IY, Huang WS, Lin YS, Kuan FC, Shu LH, Cheng YC, Yang YH, Wu CY: Danshen improves survival of patients with colon cancer and dihydroisotanshinone I inhibit the proliferation of colon cancer cells via apoptosis and skp2 signaling pathway. J Ethnopharmacol 2017;209:305-316.

5 Huang MH, Lin SZ, Lin PC, Chiou TW, Harn YW, Ho LI, Chan TM, Chou CW, Chuang CH, Su HL, Harn HJ: Brain tumor senescence might be mediated by downregulation of S-phase kinase-associated protein 2 via butylidenephthalide leading to decreased cell viability. Tumour Biol 2014;35:4875-4884. Tsai NM, Chen YL, Lee CC, Lin PC, Cheng YL, Chang WL, Lin SZ, Harn HJ: The natural compound n-butylidenephthalide derived from Angelica sinensis inhibits malignant brain tumor growth in vitro and in vivo. J Neurochem 2006;99:1251-1262.

7 Pang CY, Chiu SC, Harn HJ, Zhai WJ, Lin SZ, Yang HH: Proteomic-based identification of multiple pathways underlying n-butylidenephthalide-induced apoptosis in LNCaP human prostate cancer cells. Food Chem Toxicol 2013;59:281-288.

-8 Chiu SC, Chen SP, Huang SY, Wang MJ, Lin SZ, Harn HJ, Pang CY: Induction of apoptosis coupled to endoplasmic reticulum stress in human prostate cancer cells by n-butylidenephthalide. PLoS One 2012;7:e33742.

-9 Wei CW, Lin CC, Yu YL, Lin CY, Lin PC, Wu MT, Chen CJ, Chang W, Lin SZ, Chen YL, Harn HJ: n-Butylidenephthalide induced apoptosis in the A549 human lung adenocarcinoma cell line by coupled down-regulation of AP-2alpha and telomerase activity. Acta Pharmacol Sin 2009;30:1297-1306.

10 Chen YL, Jian MH, Lin CC, Kang JC, Chen SP, Lin PC, Hung PJ, Chen JR, Chang WL, Lin SZ, Harn HJ: The induction of orphan nuclear receptor Nur77 expression by n-butylenephthalide as pharmaceuticals on hepatocellular carcinoma cell therapy. Mol Pharmacol 2008;74:1046-1058.

11 Emerling BM, Akcakanat A: Targeting PI3K/mTOR signaling in cancer. Cancer Res 2011;71:7351-7359.

12 Younes H, Leleu X, Hatjiharissi E, Moreau AS, Hideshima T, Richardson P, Anderson KC, Ghobrial IM: Targeting the phosphatidylinositol 3-kinase pathway in multiple myeloma. Clin Cancer Res 2007;13:37713775.

13 Ying J, Xu Q Liu B, Zhang G, Chen L, Pan H: The expression of the PI3K/AKT/mTOR pathway in gastric cancer and its role in gastric cancer prognosis. Onco Targets Ther 2015;8:2427-2433. 


\section{Cellular Physiology Cell Physiol Biochem 2018;48:2231-2246

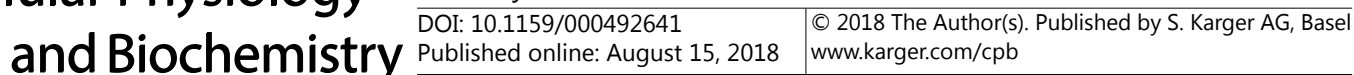

14 Riquelme I, Saavedra K, Espinoza JA, Weber H, Garcia P, Nervi B, Garrido M, Corvalan AH, Roa JC, Bizama C: Molecular classification of gastric cancer: Towards a pathway-driven targeted therapy. Oncotarget 2015;6:24750-24779.

15 DeYoung MP, Horak P, Sofer A, Sgroi D, Ellisen LW: Hypoxia regulates TSC1/2-mTOR signaling and tumor suppression through REDD1-mediated 14-3-3 shuttling. Genes Dev 2008;22:239-251.

-16 Brugarolas J, Lei K, Hurley RL, Manning BD, Reiling JH, Hafen E, Witters LA, Ellisen LW, Kaelin WG, Jr.: Regulation of mTOR function in response to hypoxia by REDD1 and the TSC1/TSC2 tumor suppressor complex. Genes Dev 2004;18:2893-2904.

17 Wullschleger S, Loewith R, Hall MN: TOR signaling in growth and metabolism. Cell 2006;124:471-484.

$\checkmark 18$ Charlson ME, Pompei P, Ales KL, MacKenzie CR: A new method of classifying prognostic comorbidity in longitudinal studies: development and validation. J Chronic Dis 1987;40:373-383.

19 Hsieh TF, Yang YW, Lee SS, Lin TH, Liu HH, Tsai TH, Chen CC, Huang YS, Lee CC: Use of 5-alpha-reductase inhibitors did not increase the risk of cardiovascular diseases in patients with benign prostate hyperplasia: a five-year follow-up study. PloS One 015;10:e0119694.

-20 Yang YW, Hsieh TF, Yu CH, Huang YS, Lee CC, Tsai TH: Zolpidem and the risk of Parkinson's disease: a nationwide population-based study. J Psychiatr Res 2014;58:84-88.

21 Lin HC, Chao PZ, Lee HC: Sudden sensorineural hearing loss increases the risk of stroke: a 5-year follow-up study. Stroke 2008;39:2744-2748.

-22 Huang da W, Sherman BT, Lempicki RA: Bioinformatics enrichment tools: paths toward the comprehensive functional analysis of large gene lists. Nucleic Acids Res 2009;37:1-13.

23 Huang da W, Sherman BT, Lempicki RA: Systematic and integrative analysis of large gene lists using DAVID bioinformatics resources. Nat Protoc 2009;4:44-57.

-24 Chen EY, Xu H, Gordonov S, Lim MP, Perkins MH, Ma'ayan A: Expression2Kinases: mRNA profiling linked to multiple upstream regulatory layers. Bioinformatics 2012;28:105-111.

25 Chiu SC, Huang SY, Chen SP, Su CC, Chiu TL, Pang CY: Tanshinone IIA inhibits human prostate cancer cells growth by induction of endoplasmic reticulum stress in vitro and in vivo. Prostate Cancer Prostatic Dis 2013;16:315-322.

-26 Hsueh KW, Chiou TW, Chiang SF, Yamashita T, Abe K, Borlongan CV, Sanberg PR, Huang AY, Lin SZ, Harn HJ: Autophagic down-regulation in motor neurons remarkably prolongs the survival of ALS mice. Neuropharmacology 2016;108:152-160.

-27 Zhang Q, Chen ZW, Zhao YH, Liu BW, Liu NW, Ke CC, Tan HM: Bone Marrow Stromal Cells Combined With Sodium Ferulate and n-Butylidenephthalide Promote the Effect of Therapeutic Angiogenesis via Advancing Astrocyte-Derived Trophic Factors After Ischemic Stroke. Cell Transplant 2017;26:229-242.

-28 Yang CS, Matsuura K, Huang NJ, Robeson AC, Huang B, Zhang L, Kornbluth S: Fatty acid synthase inhibition engages a novel caspase-2 regulatory mechanism to induce ovarian cancer cell death. Oncogene 2015;34:3264-3272.

29 Ben Sahra I, Regazzetti C, Robert G, Laurent K, Le Marchand-Brustel Y, Auberger P, Tanti JF, GiorgettiPeraldi S, Bost F: Metformin, independent of AMPK, induces mTOR inhibition and cell-cycle arrest through REDD1. Cancer Res 2011;71:4366-4372.

-30 Ellisen LW, Ramsayer KD, Johannessen CM, Yang A, Beppu H, Minda K, Oliner JD, McKeon F, Haber DA: REDD1, a developmentally regulated transcriptional target of p63 and p53, links p63 to regulation of reactive oxygen species. Mol Cell 2002;10:995-1005.

-31 Cam M, Bid HK, Xiao L, Zambetti GP, Houghton PJ, Cam H: p53/TAp63 and AKT regulate mammalian target of rapamycin complex 1 (mTORC1) signaling through two independent parallel pathways in the presence of DNA damage. J Biol Chem 2014;289:4083-4094.

-32 Shoshani T, Faerman A, Mett I, Zelin E, Tenne T, Gorodin S, Moshel Y, Elbaz S, Budanov A, Chajut A, Kalinski H, Kamer I, Rozen A, Mor O, Keshet E, Leshkowitz D, Einat P, Skaliter R, Feinstein E: Identification of a novel hypoxia-inducible factor 1-responsive gene, RTP801, involved in apoptosis. Mol Cell Biol 2002;22:2283-2293.

-33 Kaper F, Dornhoefer N, Giaccia AJ: Mutations in the PI3K/PTEN/TSC2 pathway contribute to mammalian target of rapamycin activity and increased translation under hypoxic conditions. Cancer Res 2006;66:1561-1569. 


\section{Cellular Physiology Cell Physiol Biochem 2018;48:2231-2246 \begin{tabular}{ll|l} 
DOI: 10.1159/000492641 & $\begin{array}{l}\text { O 2018 The Author(s). Published by S. Karger AG, Basel } \\
\text { www.karger.com/cpb }\end{array}$ \\
\hline
\end{tabular}}

Liao et al.: Anti-Cancer Effects of Danggui on Gastric Cancer

34 Connolly E, Braunstein S, Formenti S, Schneider RJ: Hypoxia inhibits protein synthesis through a 4E-BP1 and elongation factor 2 kinase pathway controlled by mTOR and uncoupled in breast cancer cells. Mol Cell Biol 2006;26:3955-3965.

-35 Wolff NC, Vega-Rubin-de-Celis S, Xie XJ, Castrillon DH, Kabbani W, Brugarolas J: Cell-type-dependent regulation of mTORC1 by REDD1 and the tumor suppressors TSC1/TSC2 and LKB1 in response to hypoxia. Mol Cell Biol 2011;31:1870-1884.

-36 Wolff NC, McKay RM, Brugarolas J: REDD1/DDIT4-independent mTORC1 inhibition and apoptosis by glucocorticoids in thymocytes. Mol Cancer Res 2014;12:867-877.

-37 Cai C, Yu JW, Wu JG, Lu RQ Ni XC, Wang SL, Jiang BJ: [CD133 promotes the invasion and metastasis of gastric cancer via epithelial-mesenchymal transition]. Zhonghua Wei Chang Wai Ke Za Zhi 2013;16:662-667. Chan AO: E-cadherin in gastric cancer. World J Gastroenterol 2006;12:199-203.

39 Hazan RB, Qiao R, Keren R, Badano I, Suyama K: Cadherin switch in tumor progression. Ann N Y Acad Sci 2004;1014:155-163.

40 Choi YJ, Kim N, Chang H, Lee HS, Park SM, Park JH, Shin CM, Kim JM, Kim JS, Lee DH, Jung HC: Helicobacter pylori-induced epithelial-mesenchymal transition, a potential role of gastric cancer initiation and an emergence of stem cells. Carcinogenesis 2015;36:553-563. 